J. Clin. Chem. Clin. Biochem.

Vol. 17, 1979, pp. 465-469

\title{
Steueralgorithmus für die extrakorporale Blutglucoseregulierung
}

\author{
Von J. D. Kruse-Jarres ${ }^{1}$ ), M. Bresch und U. Lehmann \\ Klinisch-Chemische und Experimentelle Laboratorien, Chirurgische Universitätsklinik Freiburg i.Br.
}

(Eingegangen am 5. Dezember 1978/9. Februar 1979)

Zusammenfassung: Der vorgestellte Regelungsmechanismus für die extrakorporale Blutglucoseregulierung beruht auf einer einfachen Proportional/Differential-Regelung. Dabei wird im Minutentakt unter Berücksichtigung von Glucoseabbau, Insulinhalbwertszeit in vivo und Reaktionsverzögerung zwischen Blutentnahme und Insulinwirkung auf den entsprechenden Blutglucosewert ein Berechnungszyklus durchgeführt, der sich in einem Kurzzeitsystem ( $1 \mathrm{~min}$ ) oder in einem Langzeitsystem ( $5 \mathrm{~min}$ und länger) entsprechend der Geschwindigkeit der Glucoseveränderung laufend wiederholt. Die Entscheidungen von seiten des Rechners fallen aufgrund von digital umgesetzten Tabellen analog zu den Kennlinien der Proportionalsteuerung und anhand von Glucoseäquivalenztabellen, die die Insulinwirkung auf die Glucose in Abhängigkeit von der Zeit wiedergeben.

\section{Algorithm for extracorporal blood glucose regulation}

Summary: A control mechanism is described, based on a simple proportional/differential regulation. The calculation takes one minute and it takes into account glucose degradation, insulin half life in vivo, and the delay between blood sampling and insulin action on the blood glucose value. This is repeated continuously every minute (short time mode) or every 5 minutes or more (long time mode), depending on the rate of change of the blood glucose. Operator decision is based on digitally converted tables, which are analogous to the graph of proportional control, and on glucose equivalent tables, which give the insulin effect on glucose as a function of time.

\section{Einleitung}

Unter der Voraussetzung, däß Insulin zwar die Glucoseassimilation fördert, jedoch im Organismus natürlicherweise und individuell abgebaut wird, läßt sịch auch dann mathematisch ein Regelkreis beschreiben, wenn vernachlässigt wird, däß weitere Einflußgrößen innerhalb gewisser Grenzen sehr variable Interventionen geltend machen können. Eịne derartige Reglerfunktion läßt sich mit den mathematischen Mitteln der Regelungstechnik finden. Dieser Weg führt jedoch zu Differentialgleichun= gen, deren Lösungen nur mit komplizierten mathematischen Formeln möglich ist. Moderne Computer aber können diese Probleme dưch itèrative Näherung umgehen und stellen somit brauchbare und sehr anschauliche Löṣungswege dar.

\footnotetext{
$\left.{ }^{1}\right)$ Mit Unterstïtżung der Deutschen Forschungsgemeinschaf̣t
} (Kr 468/7)
Nachdem Kadish (1) 1963 mittels eines einfachen automatischen Feedback-Kontrollsystems versuchte, die Blutglucosekonzentration stabil zu halten, gelang es Albisser et al. (2) 1973.erstmals, einen Steueralgorithmus einzusetzen, der befriedigende Ergebnisse brachte. Es handelt sich dabei um eine tangens-hyperbolische Funktion, mit deren Hilfe zwar ein Abfall der Blutglucosekonzentration möglich war, dies jedoch nicht ohne Gegensteuerung mit Glucagon bzw. Glucose. Ebenfalls gegensteuern mußte die zur gleichen Zeit an der Entwicklung eines künstlichen Pankreas arbeitende Arbeitsgruppe um Pfeiffer (3), deren Insulininfusionsraten als biquadratische Funktionen der Blutglucosekonzentration betrachtet werden müssen. Ziel ihrer Arbeiten wạr vor allem die auf diesem Modell beruhende Vorausberechnung von Blutglucosekonzentrationen und die entsprechend frühzeitige Reaktion. Auf die Gegenregulation mit Glucose verzichten die Algorithmen von Mirouze et al. (4). Ihre Kennlinien sind durch zwei 
verschiedene Kurven für Anstieg und Abfall der Blutglucosekonzentration gekennzeichnet.

Die Ergebnisse unserer Arbeitsgruppe beruhen auf der kontinuierlichen Blutglucoseregistrierung (5-7), mit welcher inzwischen eine mehr als 10-jährige Erfahrung vorliegt, und auf der Computer-gesteuerten Regulierung, die wir seit 1972 mit laufenden Modifikationen der Steueralgorithmen (8-11) am "aep" (artifizielles endokrines Pankreas, Abb. 1) durchführen. Bei der Entwicklung unserer Algorithmen für den geschlossenen Regelkreis (closed-loop system) haben wir uns von dem Gedanken leiten lassen, daß starre Schwellenwerteinstellungen keinen Erfolg haben und daß individuelle Blutglucosereaktionen die anwendungsstarren Regelmechanismen auf biomathematischer Grundlage wie Tangentialoder Hyperbelalgorithmen häufig durchkreuzen. Außerdem erschien uns die alternierende Infusion von Insulin und Glucose im Sollwertbereich unphysiologisch, da verbleibende Sekretionsmechanismen in vivo blockiert werden und ein unnötig hoher Mehrverbrauch an Insulin resultiert. Unser Regelungsmechanismus beruht auf einer einfachen Proportional/Differential-(P/D-)Regelung.

\section{Material und Methoden}

Grundlage unserer Steueralgorithmen sind Entscheidungstabellen, die von folgender Reglerhardware gesteuert werden:

\section{Reglerhardware}

Bei experimentellen Regelungssystemen setzen sich immer wieder digital arbeitende Regler durch, bei denen der Regelungsalgorithmus als Steuerprogramm auf einem Prozeßrechner oder auf einem Mikrocomputer realisiert werden kann (DDC - Direct Digital Control). Bei unserem Projekt wird ein für ProzeßSteuerungsanwendungen modifizierter Minicomputer (Dietz 621) mit $32 \mathrm{kB}$ Kernspeicher, einer 2,4 MB Wechselplatte und dem Plattenbetriebssystem DBOS eingesetzt. Der Regelungsalgorithmus ist als Steuerprogramm in der höheren Programmiersprache Basex formuliert, eine modifizierte Version von Basic.

Die Verwendung eines solchen, recht komfortabel atmutenden Prozessrechners richtete sich im vorliegenden Fall nach den vorhandenen Gegebenheiten. Wesentlich kleinere Versionen (z.B.

Commodore PET 2000) sind ohne Schwierigkeiten verwendbar. Doch bringt die hier vorgestellte Version außer für die Formulierung des Regelungsalgorithmus' im Hinblick auf die Datenverarbeitung noch weitere zusätzliche Vorteile für das experimentelle System mit sich:

- A/D-Wandlung der Meßwerte, Mitteilung von Meßwerten und Speicherung von Meßwerten in EDV-gerechter Form,

- Steuerung des Meßvorgangs (Abtastzeit),

- Speicherung beliebiger Standardbezugskurven,

- Speicherung von Regleraktivitäten, Errechnung und Ausgabe von Dosierübersichten und Dosierbilanzen,

- Statistische Nachbearbeitung von Meßwerten ohne erneute Umsetzung auf andere EDV-Anlagen (curve-fitting, Regressionsberechnung, Filterung, Ermittlung kinetischer Konstanten, Korrelationsberechnungen zur Ermittlung von Oszillationen),

- Warnung bei Erreichen kritischer Zustände,

- Abspeicherung von Meßwerten auf Dauerdatenträger (z.B. Lochstreifen).

Das Prozeßinterface zwischen dem Prozessor und der Insulininfusionspumpe wurde eigens von uns konstruiert und wurde an anderer Stelle ausführlich beschriẹben (12).

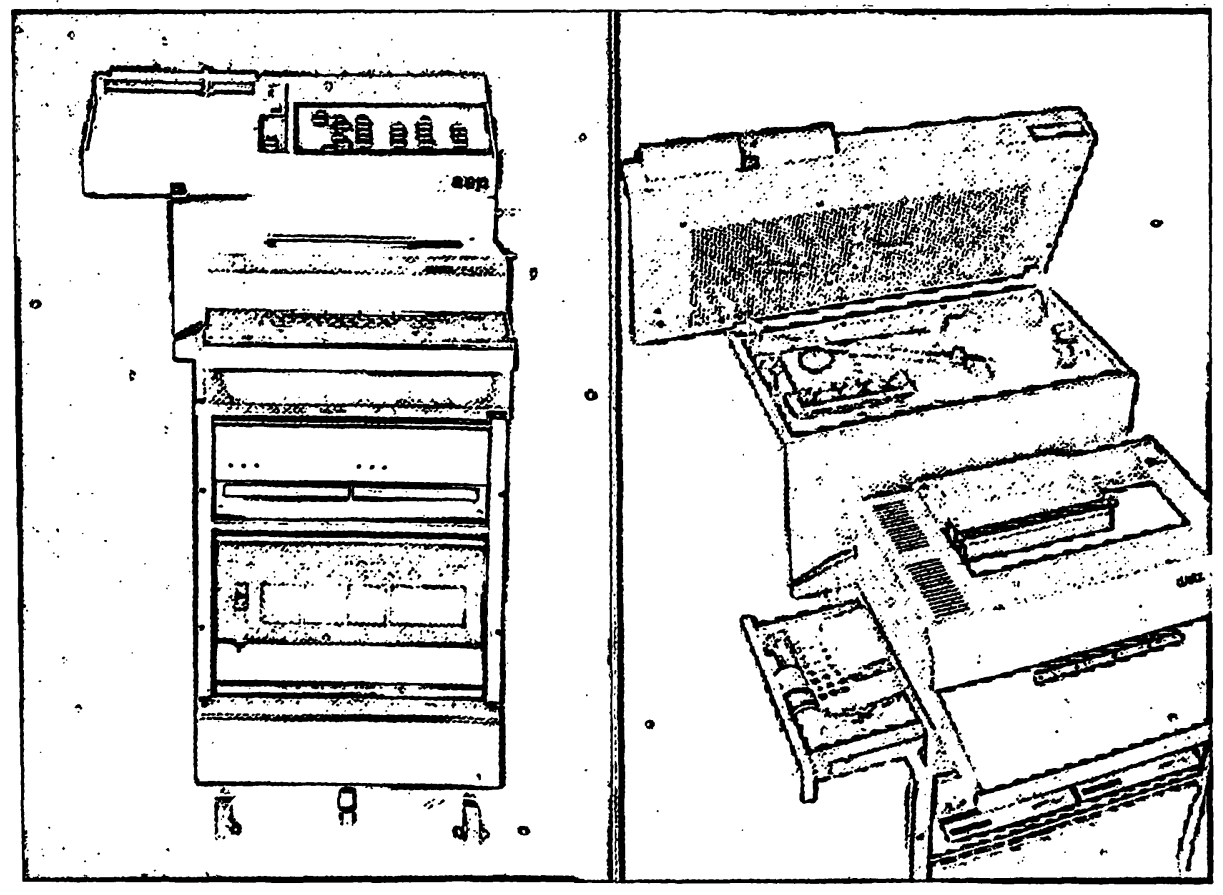

Abb. 1. Ansicht und Aufsicht des ,aep“ (artificial endocrine pancreas) mit Rechner, Dialogkonsole, Schreiber und Interface (links), sowie der analytischen Einheit nach Hochklappen von Schreiber und Interface. Die analytische Einheit mit der Abnahmenadel kann auf einer Teleskopschiene nach rechts ausgefahren werden, um den Weg vom Abnahmeort möglichst kurz zu gestalten. 


\section{Reglersoftware}

Bei Verwendung von Prozeßrechnern als Grundlage für technische Regler ist die Reglersoftware (Regelungsalgorithmus) ein Steuerungsprogramm, das in einer höheren Programmiersprache oder in einer Assemblersprache formuliert ist: Die Darstellung des Regelungsalgorithmus' in Form eines Computerprogramms erlaubt eine einfache, schnelle und übersichtliche Erstellung und Modifikation von Reglerstrukturen bzw. Regelungsgrößen. Der Algorithmus reagiert auf Regelgrößenabweichungen und auf $\ddot{A}$ nderungen der Regelgröße; beide Größen stellen den Reglerinput dar. Regleroutput sind die Steueranweisungen an die Insulinpumpe. Diese kann nur ein-bzw. ausgeschaltet werden, so daß im einfachsten Fall ein nicht-linearer Dreipunktregler vorliegt. Die Förderleistung beträgt bis zu $2 \mathrm{U}$ Insulin pro Minute. Der Steuerzustand der Pumpe wird programmintern über sog. Entscheidungstabellen bestimmt. Dabei wird im Minutentakt unter Berücksichtigung von Glucoseabbau, Insulinhalbwertszeit in vivo und Reaktionsverzug (time delay) zwischen Blutentnahme und Insulinwirkung (13) ein Berechnungszyklus durchgefürt, der sich in einem Kurzzeit- oder in einem Langzeitsystem entsprechend der Glucoseveränderung pro Zeiteinheit laufend wiederholt. Die Entscheidungen von seiten des Rechners fallen aufgrund von digital umgesetzten Tabellen (Abb. 2) analog zu den Kennlinien der Proportionalsteuerung und aufgrund von Glucoseäquivalenztabellen (13), die dic Insulinwirkung auf die Glucose in Abhängigkeit von der Zeit wiedergibt. Die Entscheidungstabellen sind zweid imensionale, diskret aufgebaute, mathematischen Wahrscheinlichkeitstabellen entsprechende Struk turen, aus deren Intervalleinteilungen eindeutige Steuerzu stände entnommen werden können. Die Intervalleinteilungen werden für die Kurzzeit- und für die Langzeitregelung unterschiedlich vorgenommen.

Das Kurzzeitsystem arbeitet auf der Basis der Minutend ifferenz von zwei aufeinander folgenden Blutglucosewerten im Bereich um den Sollwert (SW). Dies geschieht mit dem Zweck, im physiologischen Bereich auf schnclle und große Änderungen auch schnell reagieren zu können. Wenn zwischen dem Sollwert (SW)

Kurzzeitsystem (1min)

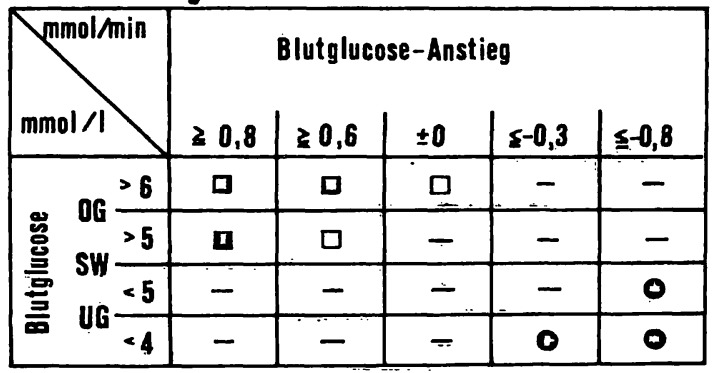

Langzeitsystem (varią̧el $;>5 \mathrm{~min}$ )

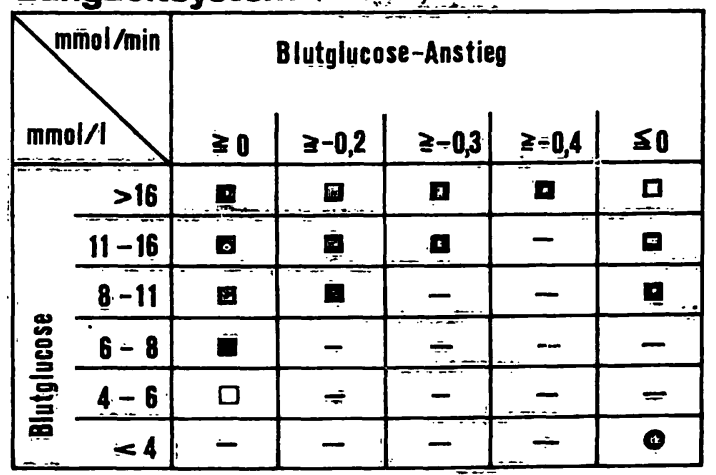

insulin (Infusionsperioden 4 min bzw. variạbel')

Insulin: (Basisssekrietion)

- Glucose (theoretische Infusion)

Abb. 2. Beispiel vọn Entscheidungstabellen mit kombinierter Schwellen/Stufen-Steueriung für die extrakorporale Blutglucoseregulierung. und dem oberen Grenzwert (OG) der Blutglucosewert um mehr als $0.8 \mathrm{mmol} / \mathrm{l} \cdot \mathrm{min}$ steigt, dann wird die Insulinpumpe für zunächst $1 \mathrm{~min}$ angesteuert d.h. es werden $2 U$ Insulin injiziert. (Desgleichen würde theoretisch eine Glucosepumpe angesteuert, wenn die Blutglucose $\mathrm{zw}$ ischen dem SW und dem unteren Grenzwert (UG) pro min um mehr als $0.8 \mathrm{mmol} / 1$ fällt.)

Während im Kurzzeitsystem die Entscheidungen minütlich aufgrund der Differenz zum Vorwert getroffen wird, werden im Langzeitsystem zwar auch minütlich alle Messungen registriert, die Entscheidungen werden jedoch individuell an Hand von Regressionsberechnungen gefällt. Um kleinere Schwankungen zu umgehen, werden die Regressionsberechnungen in Zeiträumen vorgenommen, die mindestens $5 \mathrm{~min}$, gewöhnlich jedoch 10-20 min umfassen.

\section{Resultate und Diskussion}

Der zeitliche Abstand zwischen 2 Langzeitregelungsprozessen muß so gewählt werden, daß die Wirkungstotzeit des injizierten Insulins überschritten wird, so daß sich Regleraktivitäten schon auf die Regelgröße auswirken können, bevor der Regler erneut aktiv wird. Daraus resultiert eine Zeitverzögerung von $15 \mathrm{~min}$, die die Analysenzeit von $90 \mathrm{~s}$ beinhaltet. Sie führt zwangsläufig zu Oszillationen, deren Amplitude von der Zeit zwischen Abnahme des Blutes und registrierter Wirkung der inzwischen erfolgten Insulininjektion abhängt (Abb. 3).

Die Reaktionsverzögerung in vivo und im Analysensystem produziert Oszillationen der Blutglucose, die durch hohe Insulingaben noch erheblich verstärkt werden und somit eine artifizielle Gegenregulation notwendig machen würden. Um diese unphysiologische Schaukeltherapie zu umgehen, muß es das Ziel sein, den Blutglucoseverhältnissen eine möglichst gleichmäßige Insulinsubstitution anzupassen, wie dies im Bild einer Computersimulation dargestellt ist (Abb. 4).

$\mathrm{Zu}$ einem ausgeprägten Nachschwingen von Hyperglykämien nach artifizieller Normalisierung der Blutglucose kommt es häufig infolge eines Pufferbereichs zwischen

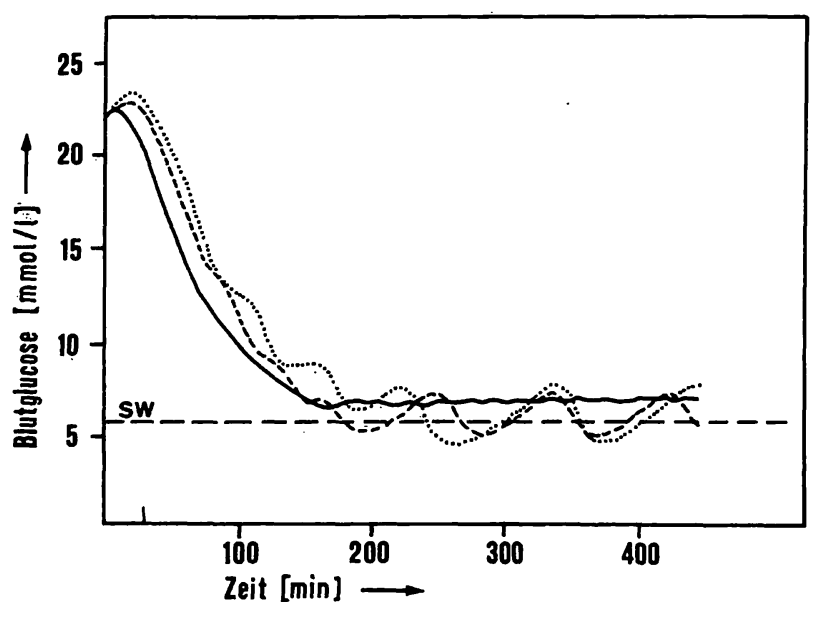

Abb. 3. Einfluß der zeitverzögerten Insulininjektion als Antwort auf das Verhalten der Blutglucose. Mit zunehmender Zeitverzögerung ( $-1 \mathrm{~min} ;---10 \mathrm{~min} ; \cdots 2 \mathrm{~min}$ ) nehmen die Amplituden der Schwingungen um den Schwellenwert (SW) zu. 


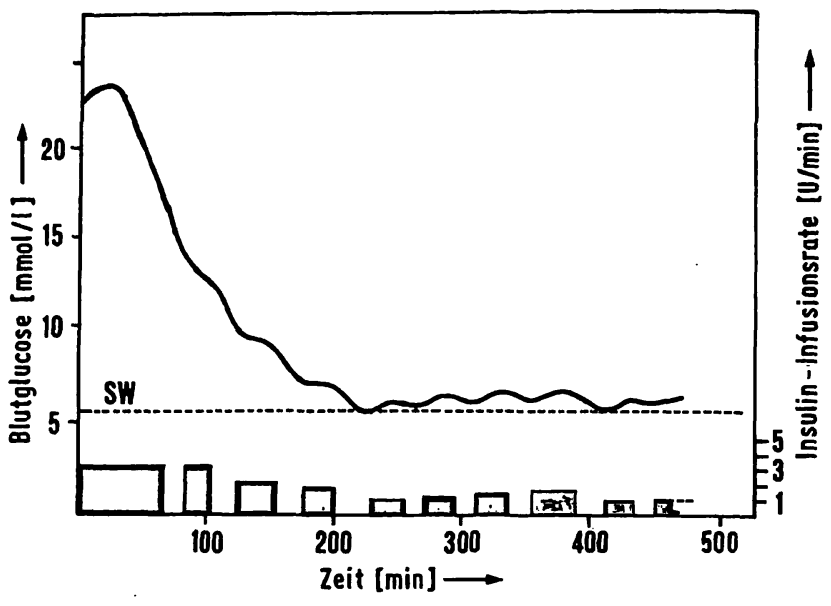

Abb. 4. Computer-Simulation einer diabetischen Stoff wechsellage unter den Bedingungen der automatischen Insulinsubstitution mit Basissekretion um den Sollwert (SW) herum.

Sollwert (SW) und oberer Grenze (OG), da hier bei langsamer Veränderung kein, dagegen bei Übertreten der OG zuviel Insulin infundiert wird. Als dämpfende Maßnahme haben wir eine Art Basissekretion im Sollbereich eingefuhrt. Diese ist bestimmt durch:

1. die Glucosezunahme im Infusionsintervall. Dazu muß folgende Bedingung erfüllt sein:

$\mathrm{St} \cdot \mathrm{t}(\mathrm{mmol} / 1 \cdot \min \cdot \min )>0,5$

$\mathrm{St}=$ Anstieg der Blutglucose $(\mathrm{mol} / \mathrm{l})$ pro $\mathrm{min}$

$\mathrm{t}=$ Zeit seit der letzten Infusion

2. die Infusionsdauer als Funktion des Abstands vom Sollwert (SW). Sie errechnet sich nach der Formel $t_{I}=\frac{10 \cdot(0,5-F) \cdot(I W-S W)}{O G-S W}+10 \cdot F$

$\mathrm{F}=$ Faktor der Basissekretion $(6-800 \mathrm{mE} / \mathrm{h})$

$\mathrm{OG}=$ obere Glucosegrenze $(\mathrm{mmol} / \mathrm{l})$

$\mathrm{SW}=$ Sollwert der Blutglucose $(\mathrm{mmol} / \mathrm{l})$

$\mathrm{IW}=$ Istwert der Blutglucose $(\mathrm{mmol} / \mathrm{l})$

und leitet sich aus folgender Graphik ab (Abb. 5).

Als Ergebnis der Einführung eines solchen 3. Regelungssystems in Form einer Basissekretion zeigt sich eine gleichmäßige Insulininfusionsrate, wobei die vorher großen Oszillationen gedämpft werden (Abb. 6).

Die Wahl unterschiedlicher Steuerzustände versetzt den Benutzer des ,aep“ (artifizielles endokrines Pankreas) in die Lage größtmöglicher Anpassung an die individuelle Situation, so daß er verschiedenen experimentellen und klinischen Bedingungen gerecht werden kann. Wird die Selektion vom Programm selbst vorgenommen, können Formen adaptiver Regelung realisiert werden. Der jeweils festgelegte Steuerzustand der Pumpen bleibt in höheren Blutglucosekonzentrationsbereichen für $1 \mathrm{~min}$ bis zum nächsten Abtastzyklus bestehen, so daß Schalttakte von

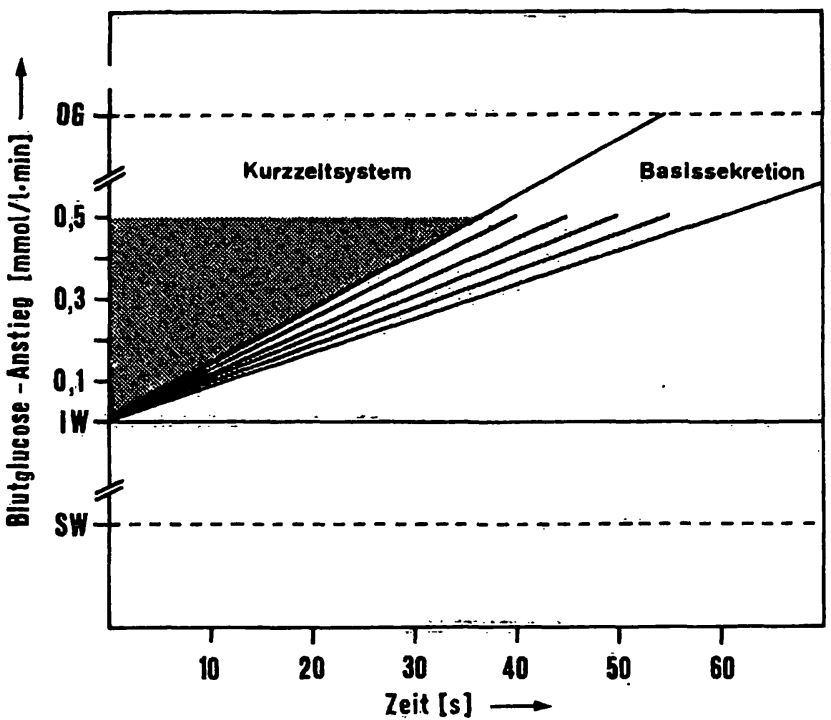

Abb. 5. Basissekretion eines Insulininfusionssystems zwischen Sollwert (SW) bzw. Istwert (IW) und oberer Glucosegrenze (OG). Die Insulin-Basissekretion erfolgt zwischen einem Blutglucoseanstieg von $0,5-0,8 \mathrm{mmol} / 1 \cdot \mathrm{min}$.

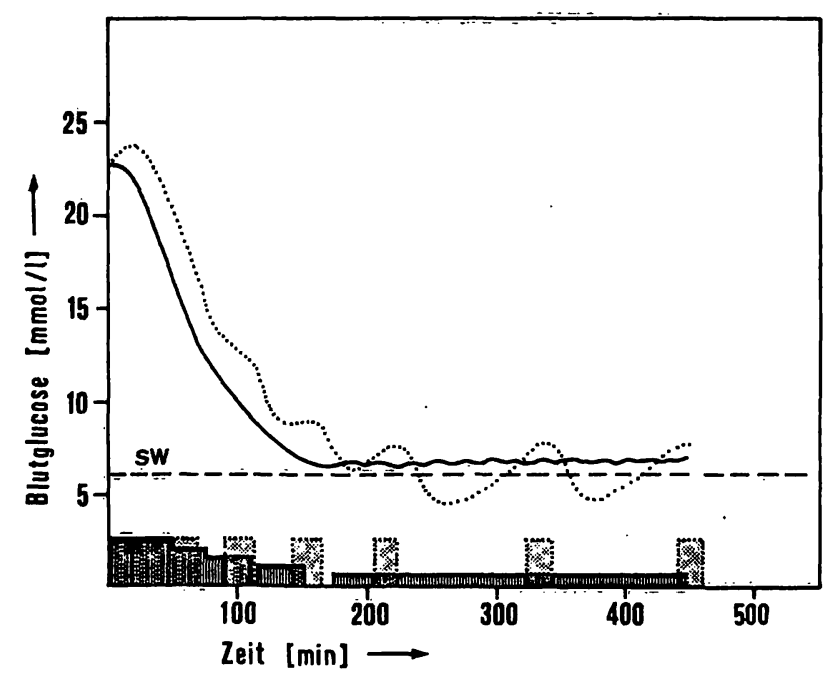

Abb. 6. Dämpfung der Oszillationen und Normalisièrung der Blutglucose infolge einer automatisierten Insulintherapie mit Basissekretion im Sollwertbereich (durchgezogene Linie bzw. schraffierte Felder) im Vergleich zur diskontinuierlichen Insulintherapie (punktierte Linie und Felder).

genau 1 min Dauer resultieren. Bei Blutglucosekonżentrationen, die nur leicht über dem oberen Grenzwert für die Insulinansteuerung liegen, werden Schalttakte von $1 / 10$ min eingelegt.

Die Entscheidungstabellen sind der eigentliche Kern des Regelungsalgorithmus', obwohl sie nur etwa 10-20\% dẹ Gesamtprogrammlänge des Steuerprogramms ausmachen. Der Rest des Programms besteht aus Organisationsanweisungen und Umformungsbefehlen (Zeitsteuerung, Umsetzung und Mittelung der Meßwerte, Konzentrationsberechnung anhand von Ștandardkurven, Verarbeitung von Benutzereingaben und voin manuell ein- 
gegebenen oder automatisch befohlenen Meßunterbrechungen). Der leichte Zugriff zu den diversen Programmen in verschiedenen Ebenen macht das gesamte System sehr flexibel und erlaubt jederzeit Modifikation im Detail.

\section{Literatur}

1. Kadish, A. H. (1963), Trans. Amer. Soc. Artif. Int. Org. 9, 363-367

2. Albisser, A. M., Leibel, B. S., Ewart, T., Davidovac, Z., Botz, C. K. \& Zingg, W. (1974), Diabetes 23, 389-396

3. Pfeiffer, E. F. \& Thum, Ch. (1974), Naturwissenschaften 61 , $455-456$

4. Mirouze, J., Selam, J. L., Pham, T. C. \& Cavadore, D. (1977) Diabetologia 13, 273-278

5. Kruse-Jarres, J. D., Reiter, J. \& Klingmüller, V. (1969),

Klin. Wochenschr. 47, 462-469

6. Kruse-Jarres, J. D., Hilpert, C. \& Klingmüller, V. (1972), Med. Klin. 67, 226-230

7. Kruse-Jarres, J. D. (1974), Intensivmed. 11, 277-283

\section{Danksagung}

An dieser Stelle sei stellvertretend für viele der direkt oder am Rande beteiligten Mitarbeiter vor allem Frau G. Broun und Herrn $R$. Naegele für ihre zuverlässige und hilfreiche Mitarbeit herzlich gedankt.

8. Kruse-Jarres, J. D. \& Geist, W. (1977), Horm. Metab. Res. Suppl. No. 7, 137-147

9. Geist, W. \& Kruse-Jarres, J. D. (1977), J. Clin. Chem. Clin. Biochem. 15, 155

10. Naegele, R. \& Kruse-Jarres, J. D. (1978), Techn. Med. 8 , $142-145$

11. Kruse-Jarres, J. D., Braun, G., Naegele, R., Bresch, M. \& Lehmann, U. (1979), Horm. Metab. Res. im Druck

12. Kruse-Jarres, J. D., Braun, G., Bresch, M., Lehmann, U. Letulé, U., Naegele, R. \& Winkelmann, R., Med. Welt 30, 83-88

13. Naegele, R. \& Kruse-Jarres, J. D. (1979), Med. Techn. 99, $67-70$
Prof. Dr. J. D. Kruse-Jarres

Klin. Chem. und Experiment. Lab. Chirurg. Univ. Klinik

Hugstetterstraße 55

D-7800 Freiburg i.Br 
